\title{
Morphologic Criteria of Lesion Activity in Neovascular Age-Related Macular Degeneration: A Consensus Article
}

\author{
Stefania Miotto, ${ }^{1}$ Nicola Zemella, Elena Gusson, ${ }^{3}$ Giacomo Panozzo, Sandro Saviano, \\ Giuseppe Scarpa, ${ }^{6}$ Giorgio Boschi, ${ }^{6}$ Stefano Piermarocchi ${ }^{7}$; \\ on behalf of the Italian GAT (Gruppo Angiografico Triveneto) Study Group
}

\begin{abstract}
Intravitreal antivascular endothelial growth factor drugs represent the current standard of care for neovascular age-related macular degeneration (nAMD). Individualized treatment regimens aim at obtaining the same visual benefits of monthly injections with a reduced number of injections and follow-up visits, and, consequently, of treatment burden. The target of these strategies is to timely recognize lesion recurrence, even before visual deterioration. Early detection of lesion activity is critical to ensure that clinical outcomes are not compromised by inappropriate delays in treatment, but questions remain on how to effectively monitor the choroidal neovascularization (CNV) activity. To assess the persistence/recurrence of lesion activity in patients undergoing treatment for $\mathrm{nAMD}$, an expert panel developed a decision algorithm based on the morphological features of CNV. After evaluating all current retinal imaging techniques, the panel identified optical coherent tomography as the most reliable tool to ascertain lesion activity when funduscopy is not obvious.
\end{abstract}

Keywords: neovascular age-related macular degeneration, choroidal neovascularization, lesion activity, morphologic criteria, antivascular endothelial growth factor

\section{Introduction}

$\mathbf{N}$ EOVASCULAR AGE-RELATED MACULAR DEGENERATION (NAMD) is a complex and progressive disease characterized by choroidal neovascularization (CNV). The prevalence of AMD, which is the most common cause of irreversible blindness in Western Countries, is expected to increase with the progressive aging of the population. ${ }^{1-4}$ In a recent metaanalysis, Wong et al. ${ }^{5}$ estimated that the number of people with AMD would rise to 196 million in 2020 and to 288 million in 2040. AMD is a debilitating and far-reaching condition, which leads to reduced quality of life and higher rates of depression. $^{6-8}$ Because AMD is a chronic disease that requires life-long monitoring, its management has become a major socioeconomic issue. 9,10
Intravitreal antivascular endothelial growth factor (antiVEGF) drugs limit moderate and severe vision loss in most nAMD patients and improve visual acuity (VA), thus representing the first-line treatment. The monthly regimen used in the MARINA and ANCHOR trials showed clear efficacy, despite the elevated treatment burden that such a schedule would entail. ${ }^{11,12}$

To limit the economic and clinical load of this therapy, physicians use a range of anti-VEGF dosing schemes for nAMD. These include "pro-re-nata" (PRN; as needed), whereby treatment is administered only when lesions are classified as active, and the "treat-and-extend" (T\&E) schedule, where the interval between administrations is gradually increased as the disease stabilizes, to maintain the lesions quiescent with the fewest possible injections. ${ }^{13}$ Another regimen is the "observe-and-plan" (O\&P),

${ }^{1}$ Department of Ophthalmology, Camposampiero Hospital, ULSS 6 Euganea, Padua, Italy.

${ }^{2}$ Department of Ophthalmology, Mestre Hospital, ULSS 3 Serenissima, Venice, Italy.

${ }^{3}$ Department of Ophthalmology, University of Verona, Verona, Italy.

${ }^{4}$ Department of Ophthalmology, Bussolengo Hospital, ULSS 9 Scaligera, Verona, Italy.

${ }^{5}$ Department of Ophthalmology, University of Trieste, Trieste, Italy.

${ }^{6}$ Department of Ophthalmology, Ca Foncello Hospital, ULSS 2 Marca Trevigiana, Treviso, Italy.

${ }^{7}$ Department of Ophthalmology, University of Padua, Padua, Italy.

(C) Stefania Miotto, et al. 2018; Published by Mary Ann Liebert, Inc. This is an Open Access article distributed under the terms of the Creative Commons Attribution License, which permits unrestricted use, distribution, and reproduction in any medium, provided the original work is properly cited. 
where the key concept is to evaluate the individual need for retreatment (after three loading doses), and then apply the optimal interval in a fixed treatment plan of a series of injections. ${ }^{14}$ Moreover, the bimonthly regimen approved for aflibercept allows to reduce the number of injections at least during the first year. ${ }^{15}$

Individualized therapy, where each patient is managed according to his/her disease characteristics, could potentially lead to a reduction in the number of intravitreal injections and patient visits, resulting in significant reduction of treatment burden. Yet, the early detection of lesion activity is critical to ensure that visual outcomes are maintained over time and not compromised by inappropriate delays.

Randomized clinical trials define the reactivation of a neovascular lesion based on specific morphological and functional criteria (Table 1), ${ }^{16-20}$ although no unanimously accepted definition of active lesion exists in clinical practice. The best-corrected visual acuity (BCVA) is currently the only globally accepted endpoint for clinical trials on AMD therapies. ${ }^{21}$ However, even in advanced disease, BCVA can remain normal until the fovea is clearly affected. Most choroidal neovascular membranes have been shown to arise eccentrically and, therefore, initially have little effect on VA. ${ }^{2}$
Muether et al. ${ }^{22}$ compared the VEGF concentration before each injection of ranibizumab with optical coherent tomography (OCT) and BCVA in AMD patients, and found that the duration of VEGF suppression ranged between 26 and 49 days. OCT evidence of CNV activity was observed after a mean of 93.7 days, while BCVA declined after a mean of 114 days. These data concluded that OCT can detect recurrence long before VA decline. ${ }^{22}$ Compared to functional parameters (eg, VA), retinal imaging techniques per se represent a sensitive and objective tool to detect signs of lesion activity and, therefore, guide the decision as to whether patients require further injections. ${ }^{22}$

The onset of a choroidal neovascular membrane can be reliably recognized by clinical examination and conventional imaging techniques. However, assessing the persistence/recurrence of lesion activity may be more challenging, as a long-standing disease determines structural changes of neurosensory retina and surrounding tissues that could mask the real progression of the disease.

The aim of this study was to perform a careful analysis of the retinal imaging techniques currently available in clinical practice, focusing on the morphological signs of persistence/ recurrence of $\mathrm{CNV}$ activity in patients undergoing anti-VEGF

Table 1. Representative Studies Using Tailored Regimens

\begin{tabular}{|c|c|c|c|}
\hline Study & $\begin{array}{l}\text { Treatment } \\
\text { protocol }\end{array}$ & Criteria for lesion activity & Instrumental follow-up \\
\hline $\begin{array}{l}\text { CATT (CATT } \\
\text { Research } \\
\text { Group et al. }{ }^{16} \text { ) }\end{array}$ & PRN & $\begin{array}{l}\text { - Fluid on OCT } \\
\text { - New or persistent hemorrhage } \\
\text { - Decreased VA compared with } \\
\text { the previous examination } \\
\text { - Dye leakage or increased lesion } \\
\text { size on FA }\end{array}$ & $\begin{array}{l}\text { - Monthly TD-OCT } \\
\text { - FA at the discretion of the } \\
\text { ophthalmologist }\end{array}$ \\
\hline $\begin{array}{l}\text { IVAN (IVAN Study } \\
\text { Investigators } \\
\text { et al. }{ }^{17} \text { ) }\end{array}$ & PRN & $\begin{array}{l}\text { - Any SRF or increasing IRF on OCT } \\
\text { - Fresh blood } \\
\text { - VA loss of } 10 \text { letters } \\
\text { - Fluorescein leakage } 25 \% \text { of the lesion } \\
\text { circumference or expansion of CNV }\end{array}$ & $\begin{array}{l}\text { - Monthly OCT } \\
\text { - Monthly fundus photo } \\
\text { - FA at the discretion of the } \\
\text { ophthalmologist }\end{array}$ \\
\hline $\begin{array}{l}\text { GEFAL } \\
\quad\left(\text { Kodjikian et al. }{ }^{18} \text { ) }\right.\end{array}$ & PRN & $\begin{array}{l}\text { - Loss of five letters with no obvious } \\
\text { atrophy or subretinal fibrosis } \\
\text { and with fluid on OCT } \\
\text { - Active exudation on OCT (SRF unless } \\
\text { stable since the last } 3 \text { monthly injections, } \\
\text { macular edema with IRF, or increase in } \\
\text { CMT of at least } 50 \mu \mathrm{m} \text { compared with } \\
\text { the previous examination) } \\
\text { - Increased CNV area or persistence } \\
\text { of leakage on FA } \\
\text { - New or persistent subretinal or intraretinal } \\
\text { macular hemorrhage }\end{array}$ & $\begin{array}{l}\text { - Monthly TD/SD-OCT } \\
\text { - Monthly fundus photo } \\
\text { - FA or and/or ICGA at the } \\
\text { investigator's discretion } \\
\text { (mandatory only at baseline } \\
\text { and } 12 \text { months) }\end{array}$ \\
\hline $\begin{array}{l}\text { HARBOR } \\
\quad \text { (Busbee et al. }{ }^{19} \text { ) }\end{array}$ & PRN & $\begin{array}{l}\text { - Five-letter decrease in VA from } \\
\text { the previous visit } \\
\text { - Any evidence of disease activity } \\
\text { on SD-OCT }\end{array}$ & $\begin{array}{l}\text { - Monthly SD-OCT } \\
\text { - FA and fundus photography } \\
\text { at baseline and at months } 3 \text {, } \\
6 \text {, and } 12\end{array}$ \\
\hline $\begin{array}{l}\text { LUCAS } \\
\text { (Berg et al. }{ }^{20} \text { ) }\end{array}$ & $\mathrm{T} \& \mathrm{E}$ & $\begin{array}{l}\text { - Any fluid on OCT } \\
\text { - New or persistent hemorrhage } \\
\text { - New or persistent dye leakage, } \\
\text { or increased lesion size on FA }\end{array}$ & $\begin{array}{l}\text { - Monthly TD or SD-OCT } \\
\text { until there were no signs of } \\
\text { disease activity on OCT } \\
\text { and biomicroscopic fundus } \\
\text { examinations, then before } \\
\text { every scheduled injection } \\
\text { - FA allowed to aid in } \\
\text { retreatment decisions }\end{array}$ \\
\hline
\end{tabular}

CMT, central macular thickness; CNV, choroidal neovascularization; FA, fluorescein angiography; ICGA, indocyanine green angiography; IRF, intraretinal fluid; OCT, optical coherence tomography; PRN, pro re nata; SD-OCT, spectral domain-OCT; SRF, subretinal fluid; T\&E, treat and extend; TD-OCT, time domain-OCT; VA, visual acuity. 
treatment. The information provided by each imaging technique was also compared, to simplify the diagnostic process through the development of an image-based algorithm.

\section{Methods}

The Delphi method is a structured communication procedure designed for building consensus from experts in a field through iterations of a survey and anonymous feedback. $^{23}$ In this study, a modified Delphi technique was used, which was based on a series of three consecutive face-toface meetings led by a moderator.

This study has been conducted by the members of an Italian scientific society (Gruppo Angiografico Triveneto or GAT) founded 30 years ago to promote the knowledge on retinal diagnostics among ophthalmologists. The GAT study group comprises eight experts coming from three universities and four hospitals. Although all members of the panel are interested in retinal diseases, they hold different tasks in their corresponding ophthalmologic institutions, which range from retinal diagnostics to laser/pharmacological treatment of retinal diseases and vitreoretinal surgery.

An English-language literature search using the PubMed Library was initially performed, focusing on specific topics inherent to the AMD, using the following keywords: "grading," "histology," "fluorescein angiography," "indocyanine green angiography," "spectral domain OCT," "OCT angiography," and "anti-VEGF." This was followed by a manual search of references cited in selected articles published in peer-reviewed journals.

Each member was assigned a specific topic that was presented to the entire group during the first meeting. During the second round, the participants presented a collection of clinical cases, questioning about advantages and disadvantages of each retinal imaging technique in the clinical decision-making process. During the third conference, the moderator proposed an anonymous survey made up of multiple-choice questions to identify key elements for the discussion, focusing on the assessment of response to anti-VEGF and the sensitivity and specificity of each imaging technique. The hypothesis catalogue that was used to guide the debate is shown in Supplementary Table S1 (Supplementary materials are available online at www.liebertpub.com/jop). The results of the debate were summarized in a treatment algorithm and approved unanimously by all members of the working group.

This article does not include original data from clinical trials conducted by any of the authors.

\section{Results}

The imaging tools currently used for the diagnosis and follow-up of nAMD patients comprise color fundus photography (CFP), fluorescein angiography (FA) and indocyanine green angiography (ICGA), and OCT and OCT angiography (OCT-A).

\section{Color fundus photography}

CFP is the closest imaging modality to correlate with clinical ophthalmoscopy and, until recently, was the gold standard for grading and staging in AMD clinical trials. ${ }^{24,25}$ At the time of nAMD diagnosis, the CFP features indicating the presence of a CNV are as follows: serous detachment of the sensory retina, hemorrhage, retinal pigment epithe- lial detachment (non-drusenoid type), fibrous tissue, or hard exudates. ${ }^{26}$ Amoaku et al. ${ }^{27}$ consider evidence of disease activity as the presence of significant new hemorrhage or exudates. However, when monitoring patients undergoing anti-VEGF treatment, only the appearance of a new retinal or subretinal hemorrhage is a reliable marker of disease activity. Indeed, the presence of hard exudates is a secondary phenomenon occurring when the extracellular fluid that accumulates within the retina is reabsorbed into the retinal capillaries. $^{28}$

\section{Fluorescein angiography}

CNV typically leaks on FA. The FA leakage patterns of $\mathrm{CNV}$ are classified as classic or occult and correlate generally with the anatomic classification, with occult membranes analogous to type $1 \mathrm{CNV}$ (located beneath the pigment epithelium) and classic membranes similar to type $2 \mathrm{CNV}$ (located in the subretinal space). ${ }^{29}$ The histopathological features determining the angiographic behavior of CNV are the vessel caliber (larger for type $2 \mathrm{CNV}$, which increases the amount of leakage) and the consistency of surrounding fibrocellular tissue (thicker in type $1 \mathrm{CNV}$, which obscures the neovascular lesion). ${ }^{30,31}$

Besides these two subtypes of CNV, retinal angiomatous proliferation (RAP; type $3 \mathrm{CNV}$ ) and idiopathic polypoidal choroidal vasculopathy (IPCV) have been recognized as peculiar forms of nAMD.

RAP refers to the development of new vessels extending outward from the neurosensory retina, with evident or occult anastomosis with the choroidal circulation. ${ }^{32}$ However, the identification of RAP can be difficult as FA commonly detects occult or minimally classic $\mathrm{CNV},{ }^{33}$ often associated with intraretinal accumulation of fluorescein in a petaloid pattern. ${ }^{34}$ IPCV was first described by Yannuzzi et al. ${ }^{35}$ as polypoidal, subretinal, vascular lesions associated with serous and hemorrhagic detachments of the retinal pigment epithelium (RPE). FA is not as useful in imaging IPCV as it is in types 1 and 2 or even type 3 neovascularization, since most patients with IPCV have fundus hyperpigmentation or serosanguineous complications that may obscure the characteristics of the polypoidal choroidal vascular abnormalities. ${ }^{36}$

Another finding in nAMD patients is the pigment epithelium detachment (PED), which represents the separation of the RPE from the underlying Bruch's membrane. It can be classified as serous PED (sPED) or fibrovascular PED (fPED). On FA, sPED shows a rapid, bright, and uniform filling of the entire lesion, reaching a peak in very late frames without leakage. sPED has sharply delineated margins, caused by the tight adherence of RPE to the Bruch's membrane. sPED could be avascular, if it is associated with disruption of the hydrophobic barrier between RPE and choriocapillaris, ${ }^{37}$ or it could be associated with occult CNV (appearing as a notching at the border of PED). ${ }^{38}$ sPED is also a prominent feature of subtypes of nAMD such as IPCV and RAP lesions. ${ }^{37}$

fPED is a subset of occult CNV and is defined as an area of irregular elevation corresponding to granular hyperfluorescence, which emerges within 1 to $2 \mathrm{~min}$ from fluorescein injection, with persistent staining or leakage in late frames. ${ }^{39}$ The management of patients with PED secondary to neovascular AMD is controversial and no consensus treatment guidelines are yet available. Traditionally, 
clinicians did not consider PED in the decision algorithms for retreatment, and none of the large-scale, prospective trials have included PED in their retreatment criteria. The VA after anti-VEGF therapy may not depend on the response of PED, but seems to be influenced by the response of other exudative findings, such as fibrin, hemorrhage, subretinal fluid (SRF), and intraretinal fluid (IRF) ${ }^{40}$

The accumulation of serous fluid beneath or within the neurosensory retina is considered a biomarker of disease activity and appears on angiograms as dye leakage or dye pooling in a petaloid pattern. As CNV growth is associated with deep structural changes involving choriocapillaris, RPE, and neurosensory retina, distinguishing leakage from fluorescein staining on FA can be challenging, especially after several treatments. ${ }^{41,42} \mathrm{CNV}$ may not exhibit fluorescein leakage if it has undergone involution (spontaneous or induced by anti-VEGF therapy) or it is enveloped by RPE proliferation. ${ }^{32}$

\section{Indocyanine green angiography}

ICGA allows the direct visualization of both classic and occult CNV. Moreover, it is employed to identify and differentiate specific subtypes of choroidal neovascular lesions, as the higher binding affinity of indocyanine green to plasma proteins leads to a slower rate of leakage from the choriocapillaris compared to fluorescein. ${ }^{43}$

ICGA is very helpful in imaging RAP branching, showing anastomotic retinal vascular communications between retina and choroid. Another ICGA finding is the late-phase staining of fibrin, which may be present in the cystic spaces. ${ }^{44}$ According to some authors, RAP can be classified into three stages: intraretinal neovascularization (stage 1), subretinal neovascularization with retinal anastomosis (stage 2), and CNV with retinal-choroidal anastomosis (stage 3). ${ }^{44}$ The progression from one stage of the disease to another is considered an important sign of activity, and, indeed, stage $3 \mathrm{RAP}$ is associated with outer retina destruction and RPE detachment. ${ }^{45-47}$

IPCV primarily involves the inner choroidal vasculature and, therefore, it is better visualized using ICGA. ${ }^{48}$ ICGA should be performed to diagnose IPCV when routine ophthalmoscopic examination indicates a serosanguineous SRF, spontaneous massive subretinal hemorrhage, notched or hemorrhagic PED, and poor response to anti-VEGF therapy. ${ }^{49}$

There are some ICGA characteristics, peculiar to polypoidal lesions that need to be investigated to clarify whether the lesion is active and, thus, requires treatment. In the late phase of ICGA, the lesion core may become hypofluorescent because of the washout of the dye, producing a ring-like staining of polyps. This may help to distinguish between polyps that are actively leaking (visible as a ring of hyperfluorescence) and those that are not (hypofluorescent ring). ${ }^{50}$ The total lesion size for IPCV is the area comprising all polyps and their branching vascular network (BVN) as shown by ICGA. Frequently, it is possible to observe pulsatile filling of the polyp in dynamic ICGA as a sign of IPCV (level 3 evidence). ${ }^{51}$

The initial goal of treatment of active IPCV is the regression of polyps as observed by ICGA: all IPCV lesions (polyps plus BVN) need to be treated as indicated by ICGA for treatment-naive patients. ${ }^{51,52}$ Incomplete regression of polyps following initial anti-VEGF treatment is not uncommon and can be assessed by ICGA at the 3-month visit.
Contrary to FA, sPED appears as a hypofluorescent lesion during all phases of ICGA. Moreover, ICGA allows to visualize the presence of an associated CNV and characterize the precise subtype (occult CNV, RAP, or IPCV). ICGA also provides a more accurate definition of fPED, allowing a better appreciation of its precise nature. ${ }^{39}$

\section{Optical coherence tomography}

The expanding use of anti-VEGF agents has led to a significant increase in the number of follow-up control visits and OCT, a quick and noninvasive diagnostic technique, has become the method of first choice in daily clinical practice. The neovascular AMD findings recognizable with spectral domainOCT (SD-OCT) may be classified based on their reflectivity. SRF and IRF are generally hyporeflective, but other findings associated with choroidal new vessels, including hemorrhage, lipid exudates, pigment clumping, subretinal fibrosis, and CNV itself demonstrate hyperreflectivity. ${ }^{53}$

SRF and IRF are considered signs of CNV activity in both naive and treated patients. SRF is defined as an elevation of the neurosensory retina and, on OCT, it appears as an area of low reflectivity between the outer surface of the retina and the RPE. ${ }^{54,55} \mathrm{SRF}$ is associated with all lesion types and is typically the first exudative sign in type 1 lesions. IRF may determine diffuse retinal thickening or cystoid space formation. ${ }^{41}$ Intraretinal cystoid fluid is a sign of a more aggressive lesion type and late presentation of chronic occult CNV.

Massive IRF exudation is often associated with lipid exudates. They are commonly seen in the outer retina, but may be scattered throughout all retinal layers and, in some cases, may cluster to form confluent plaques, particularly in patients with RAP. ${ }^{56}$

Hyporeflectivity above the RPE or between retinal layers does not necessarily mean lesion activity, as some hyporeflective lesions visible on OCT scans are not associated with fluorescein leakage. A recent OCT finding called "wedgeshaped subretinal hyporeflective lesions" should be distinguished from SRF, as its presence does not require retreatment. ${ }^{57}$ This lesion is typically located in areas of geographic atrophy in AMD eyes. Moreover, the retina overlying these hyporeflective lesions is characterized by the absence of an outer nuclear layer (ONL), the external limiting membrane, the ellipsoid zone, the interdigitation zone, and the RPE. ${ }^{57}$ Similarly, not all IRF on OCT are caused by active CNV, and it is important to differentiate between cystoid fluid caused by exudative CNV (exudative IRC) and that associated with neurosensory degeneration (degenerative IRC).

Exudative IRCs are relatively large circular or ovoid spaces, often overlying pigment epithelial detachments (type 1 or 3 lesions) or neovascular tissue (type $2 \mathrm{CNV}$ ). ${ }^{42}$ Degenerative IRF is characterized by small cystoid spaces, usually located in the inner nuclear layer of the retina, and is not associated with angiographic leakage. Moreover, on OCT, pseudocysts are not associated with retinal thickening, which differentiates them from exudative IRF. ${ }^{58}$ Retinal pseudocysts should be further distinguished from outer retinal tubulations (ORTs), which can be observed in the ONL and correspond to degenerating photoreceptors arranged in a circular or ovoid manner. ${ }^{59}$

PEDs have traditionally been classified based on clinical findings, FA and ICGA, but OCT has introduced new qualitative parameters. According to their different etiology 
(nAMD, IPCV, presumed ocular histoplasmosis, and central serous chorioretinopathy), three subtypes of PED may be identified based on the reflectivity of the material under the RPE: hollow (primarily hyporeflective), solid (primarily hyperreflective), and mixed (combination of solid and hollow PEDs). ${ }^{60}$ In nAMD patients, hollow PEDs showed the best response to anti-VEGF, whereas solid PEDs showed the poorest response. ${ }^{60}$ This suggests that the likelihood of PED flattening is inversely related to its reflectivity. Thus, in nAMD patients, we could consider hollow PEDs as possible signs of lesion activity, as they could have a good response to other intravitreal injections.

\section{Optical coherence tomography angiography}

OCT-A allows the noninvasive visualization of retinal and choroidal vascular flow by motion-contrast imaging. ${ }^{61} \mathrm{By}$ this instrument, the CNV subtypes are defined according to the position of the vascular decorrelation signal: in type 2 , it is located immediately above the RPE, and in type 1, below the RPE or between the Bruch's membrane and the RPE layers. ${ }^{62}$

A variety of CNV morphologies have been described, including the "medusa" shape, "seafan" shape, "glomerular" shape, and the "dead tree" appearance. ${ }^{63,64}$ Yet, the clinical relevance of these morphologic variations remains controversial, since they have not yet been definitely correlated to $\mathrm{CNV}$ activity. ${ }^{65}$ A study conducted by Coscas et al. ${ }^{62}$ found a correlation between some OCT-A features and $\mathrm{CNV}$ needing prompt treatment, including the abundance of branching, the presence of vascular anastomoses and loops, the presence of a peripheral arcade of the vessel termini, and the existence of a perilesional hypointense halo.

It has been hypothesized that anti-VEGF treatment may prune smaller vessels in the neovascular lesion, reducing the overall lesion size. ${ }^{66}$ Indeed, some studies focused on a quantitative analysis of $\mathrm{CNV}$, considering mainly lesion size and automated flow analysis to obtain data regarding lesion activity. ${ }^{67,68}$ However, the measurement reproducibility is challenging and image analysis algorithms have not been adequately validated. ${ }^{65}$

The ability to perform depth-resolved analysis to determine the level of the lesion could be a significant advantage of OCT-A in characterizing RAP lesions, although experience with OCT-A in RAP is still limited. ${ }^{69}$ Regarding the lesion activity assessment, a recent study evaluated the response of type 3 lesions to anti-VEGF therapy and demonstrated a decrease in lesion size. ${ }^{70}$

The motion rate of scattering particles, such as erythrocytes, in IPCV lesions depends on the polyp size: OCT-A is more suitable to detect larger polyps in which blood speed is higher, whereas small polyps may not be identified. Moreover, the faster flow velocity at the periphery of large polypoidal lesions makes them appear as rings. On the other hand, OCT-A seems highly effective in tracing the BVN of IPCV. ${ }^{71,72}$

No studies are available regarding the OCT-A features of treated polypoidal lesions. Still, it has been hypothesized that, in treated patients, shrinkage of the polyps could hamper their identification. ${ }^{72}$

\section{Discussion}

Patients undergoing intravitreal injections are followed up according to their treatment schedule. The morphologic response of neovascular lesions to anti-VEGF therapy may be monitored with all the aforementioned imaging techniques, even though the information regarding the lesion activity obtained with different tools may be contrasting or disputable.

We performed a comparative analysis of the imaging techniques to develop a treatment algorithm. One possible limitation of our study might come from the lack of geographic diversity of the board. However, we believe that the scientific background and the specific clinical exposure of each member of the panel guarantee comprehensive viewpoints to meet the aims of the study.

\section{Color fundus photography}

CFP was the basis of studies performed before the advent of more recent imaging instruments. Indeed, the continued use of CFP in current clinical trials allows the comparison with data from past studies. Mokwa et al. ${ }^{73}$ reported that, compared to FA and SD-OCT, CFP had a lower sensitivity in detecting CNV activity. However, the appearance of new retinal or subretinal hemorrhages, representing active lesions, may not be detectable on OCT. ${ }^{27}$

\section{FA and ICGA}

Traditionally, FA has been considered the reference standard to detect nAMD activity. Yet, this is particularly challenging after anti-VEGF/photodynamic therapy, as superimposed hypo/hyperfluorescence due to scarring or atrophic changes can mask dye leakage. ICGA may integrate FA findings, especially for RAP. Indeed, FA is useful to identify early-stage RAP, but, in advanced stages, fluorescein stains the whole vascular network and exudative process, resulting in an occult-like pattern.

ICGA is able to delineate abnormal vessel structure and determine the nature of the indistinct hyperfluorescence seen on FA. ${ }^{30}$ Morphologic response to treatment of IPCV lesions is usually best appreciated with ICGA, but, sometimes, the complete regression of polyps detected by ICGA is associated with persistant leakage on FA and clinical or OCT signs of activity. ${ }^{48,51}$

\section{Optical coherence tomography}

First employed in the 1990s, OCT is the most frequently used tool in the daily practice management of nAMD. The recent European Society of Retina Specialists (EURETINA) guidelines recommend to monitor disease activity monthly using SD-OCT. ${ }^{14}$

IRF or SRF may be present despite regular treatment. In the PrONTO study, 2/40 eyes $(5 \%)$ receiving a PRN regimen needed monthly retreatment, whereas results of the CATT trial showed fluid persistence (including sub-RPE fluid) at the 12 -month visit in $53 \%$ of patients assigned to the monthly treatment arm. ${ }^{16,74}$

The central retinal thickness (CRT) increase has been widely used as a retreatment criterion in many clinical trials. However, a recent review showed the poor reproducibility of CRT values due to segmentation errors, poor fixation, and use of different OCT devices. Moreover, CRT includes a medley of different retinal compartments and, therefore, is not able to distinguish variations at the level of individual pathological components. $^{41}$ 
Although conventional doses of anti-VEGF agents may reduce the associated hemorrhage, IRF, or SRF, vascularized PED (vPED) does not typically resolve. ${ }^{75}$ The reasons for the poor response of vPED to anti-VEGF therapy remain unknown. A recent study by Inoue et al. ${ }^{76}$ evaluating the prognosis of nAMD patients after intravitreal anti-VEGF therapy according to the PED subtype, showed better results in subjects with SPED. The management of patients with PED secondary to AMD is controversial and no consensus treatment guidelines are currently available as previously reported. ${ }^{40}$ However, quantitation of the change in retinal PED volume and area with SD-OCT may be useful to determine when to retreat AMD patients. ${ }^{77}$

FA still plays a key role in the initial diagnostic process, since OCT and FA examine different aspects of IRF dynamics. OCT focuses on static anatomical features (retinal thickening and the presence of SRF and IRF), while FA addresses more dynamic aspects of fluid leakage and, as a result, their findings do not always agree. Scan rates and resolution parameters of OCT have improved over the past decade and continue to develop. If OCT could accurately detect reactivation of nAMD, then FA would not be necessary. ${ }^{78}$

A number of studies have examined the relationship between OCT findings and fluorescein patterns in nAMD. In these studies, classic CNVs are associated with significantly greater volumes of subretinal hyperreflective material than occult $\mathrm{CNVs}$, which are more commonly associated with PED. ${ }^{56}$

On OCT, active RAP lesions are typified by frank cystoid macular edema overlying an fPED and associated with SRF. ${ }^{56}$ SD-OCT could provide more accurate information on the location of the lesion, identifying areas of intraretinal neovascularization and activity of the disease (PED, IRF). ${ }^{79}$
Although ICGA is essential in the initial diagnostic process, the follow-up of RAP lesions could be effectively performed with OCT. IRF or SRF accumulation is, by itself, sufficient to define the lesion as active. Coscas et al. reported that eyes with a baseline PED showed a lower mean improvement in VA, but there is no consensus on whether PED in the absence of IRF or SRF or leakage on FA should be considered a sign of lesion activity. ${ }^{80}$

IPCV may be considered active if there is clinical, OCT, or FA/ICGA evidence of any of the following features attributable to IPCV: SRF or IRF, PED, subretinal or sub-RPE hemorrhage, or fluorescein leakage. ${ }^{51}$

OCT could be more suitable to detect IPCV by recognizing abnormalities that surround the polypoidal lesions. Moreover, PED on the OCT often correspond to polypoidal lesions detected by ICGA. ${ }^{81}$ Furthermore, it is possible to identify polypoidal structures beneath RPE and attached at the back surface of RPE by high-resolution OCT scans. ${ }^{36}$

The diagnostic precision of these imaging techniques has been compared in a number of studies (Table 2$)^{78,82-94}$ : on average, OCT shows a high sensitivity (up to 100\%) in detecting lesion activity although specificity seems low.

\section{Optical coherence tomography angiography}

OCT-A has been conceived as a dye-free alternative to conventional angiography. It offers a static image of the vasculature, allowing a sharp visualization of microvascular details that are usually obscured by dye leakage or pooling on FA or ICGA. However, compared with standard angiography, the usefulness of OCT-A seems still limited by its small field of view $(3 \times 3 \mathrm{~mm}$ or $6 \times 6 \mathrm{~mm})$ and by the high chance of artifacts, including segmentation errors, signal blockage, motion artifacts, and projection artifacts. ${ }^{65}$

Table 2. Overview of Data on the Agreement Between Optical Coherence Tomography and Angiography

\begin{tabular}{|c|c|c|c|c|c|c|}
\hline Study & $\begin{array}{c}\text { Eyes/ } \\
\text { patients (n) }\end{array}$ & CNV status & $O C T$ & $\begin{array}{l}\text { Agreement FA-OCT } \\
\text { based on }\end{array}$ & $\begin{array}{c}\text { Sensitivity } \\
(\%)\end{array}$ & $\begin{array}{c}\text { Specificity } \\
(\%)\end{array}$ \\
\hline \multirow[t]{2}{*}{ Salinas-Alamán et al. ${ }^{82}$} & 62 eyes & Naive PDT- $t$ & TD & SRF, IRF & 97 & n.a. \\
\hline & & & TD & SRF, IRF & 96 & 59 \\
\hline Sandhu and Talks ${ }^{83}$ & 131 eyes & Naïve & TD & CNV, SRF, IRF, PED & 96 & 66 \\
\hline Eter and Spaide ${ }^{84}$ & 60 eyes & PDT-t & TD & SRF, IRF & 80 & 80 \\
\hline \multirow{5}{*}{ Van de Moere et al. ${ }^{85}$} & 121 eyes & PDT-t & TD & SRF & 41 & 83 \\
\hline & & & & IRF & 83 & 53 \\
\hline & & & & Gross CMO & 23 & 96 \\
\hline & & & & Solitary foveal cyst & 13 & 75 \\
\hline & & & & CRT & 63 & 82 \\
\hline van Velthoven et al. ${ }^{86}$ & $30 \mathrm{pts}$ & PDT-t & TD & CMO, SRF, CRT & 65 & 43 \\
\hline Talks et al. $^{87}$ & $134 \mathrm{pts}$ & Naive & TD & Findings of nAMD & 100 & 65 \\
\hline Malamos et al. ${ }^{88}$ & $37 \mathrm{pts}$ & Naive & SD & CNV, SRF, IRF, PED, CRT & n.a. & n.a. \\
\hline \multirow[t]{3}{*}{ Henschel et al. ${ }^{89}$} & $14 \mathrm{pts}$ & PDT-t & TD & SRF & 71 & 70 \\
\hline & & & & IRF & 90 & 40 \\
\hline & & & & SRF, IRF & 97 & 37 \\
\hline \multirow{2}{*}{ Khurana et al. ${ }^{90}$} & 59 eyes & IV-t & TD & SRF, IRF & 59 & 63 \\
\hline & & & SD & SRF, IRF & 90 & 47 \\
\hline \multirow[t]{2}{*}{ Giani et al. ${ }^{91}$} & $93 \mathrm{pts}$ & Naïve & SD & SRF & 68 & 88 \\
\hline & & & & SRF, IRF, PED & 94 & 27 \\
\hline Padnick-Silver et al. ${ }^{92}$ & $79 \mathrm{pts}$ & Naïve & TD & SRF, IRF, PED & 80 & 93 \\
\hline Do et al. ${ }^{93}$ & $87 \mathrm{pts}$ & Naive & $\mathrm{TD}$ & SRF, IRF, CRT & 69 & 57 \\
\hline Wilde et al. ${ }^{94}$ & $411 \mathrm{pts}$ & Naive & $\mathrm{SD}$ & SRF, IRF, PED & 100 & 80 \\
\hline Castillo et al. ${ }^{78}$ & $400 \mathrm{pts}$ & Mixed & $\mathrm{TD} / \mathrm{SD}$ & Meta analysis & 85 & 48 \\
\hline
\end{tabular}

CMO, cystoid macular edema; CRT, central retinal thickness; IV-t, IV treated; n.a., not applicable; nAMD, neovascular AMD; PED, pigment epithelium detachment; PDT-t, PDT-treated; pts, patients. 
FIG. 1. Flow chart for clinical follow-up based on morphological features of CNV in patients undergoing treatment for neovascular age-related macular degeneration. $\mathrm{CNV}$, choroidal neovascularization; IRF, intraretinal fluid; OCT, optical coherence tomography; RPE, retinal pigment epithelium; sPED, serous pigment epithelium detachment; SRF, subretinal fluid.

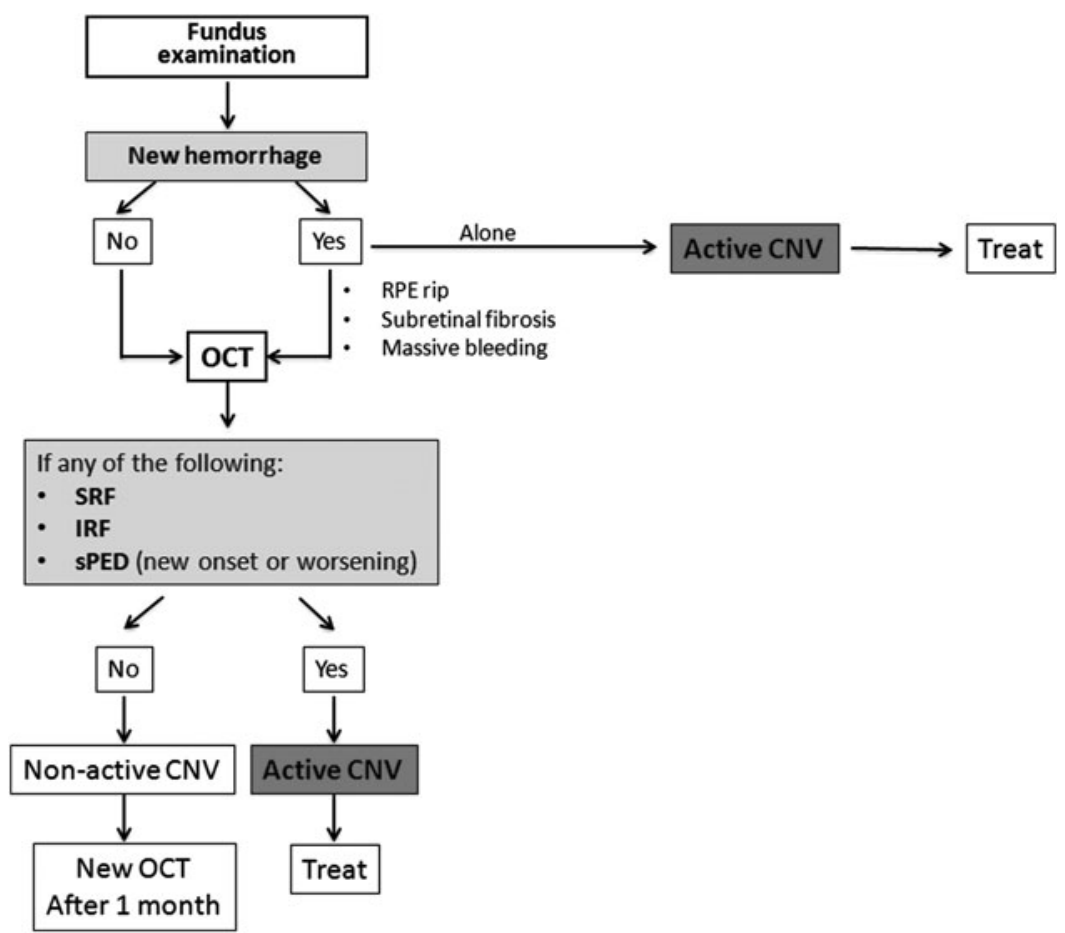

Compared to FA, OCT-A has been reported to be reliable in detecting CNV: the sensitivity and specificity of OCT-A were reported to be $50 \%$ and $91 \%$, respectively, in one study ${ }^{95}$ and $86.5 \%$ and $67.6 \%$, respectively, in another one ${ }^{96}$

ICGA is especially useful to detect polypoidal lesions. Compared to ICGA, OCT-A has been shown to be able to more clearly visualize BVNs associated with IPCV. Still, OCT-A is not able to detect polyps with the same accuracy of ICGA, as, in small lesions, the blood speed is too low to generate a detectable decorrelation signal. ${ }^{71,72,97}$

Some investigators have proposed OCT-A as a suitable alternative to FA. Yet, the vascular patterns described on OCT-A have not yet been correlated with lesion activity, ${ }^{65}$ and there are no specific OCT-A imaging biomarkers that seem to correlate with the degree of leakage visible on conventional FA. ${ }^{98}$

OCT-A is almost an upgraded OCT and appears able to integrate the structural data achievable by OCT. Indeed, Inoue et al. ${ }^{98}$ found that OCT-A, coupled with structural OCT, attained a better detection of type $1 \mathrm{CNV}$ compared to FA alone or OCT-A alone.

OCT-A may potentially translate into a reduced need for FA in clinical settings; nevertheless, OCT-A seems to be necessarily complementary to structural OCT, as a clinical endpoint indicating CNV quiescence has not been identified. ${ }^{65}$

Moreover, there are several patient- and disease-related factors that are known to preclude the reliable visualization of NV using OCT-A, and the utility of OCT-A for managing $\mathrm{NV}$ remains unclear. ${ }^{64}$

\section{Consensus Statement}

After considering all the available evidence, the panel unanimously agreed upon an OCT-based algorithm to detect lesion activity in patients with nAMD and schedule retreatment strategies with intravitreal anti-VEGF (Fig. 1). A
$100 \%$ agreement was made possible because each controversial point (eg, role of PED and usefulness of CFP alone) was discussed thoroughly based on objective data reported in the published literature.

\section{Conclusions}

Although predicting the clinical response of an eye to anti-VEGF therapy is not possible yet, it has been demonstrated that, for each patient, the duration of the anti-VEGF effect after every injection appeared quite stable over up to 3 years. $^{99}$ Therefore, the precise quantification of each patient's personal disease-free interval, strictly dependent from a prompt recognition of persistence/recurrence of $\mathrm{CNV}$ activity, is a fundamental requirement for the success of the PRN, T\&E, and O\&P protocols. ${ }^{16-20}$

An OCT-based algorithm appears the quickest and most effective way to guide the decision of the treatment schedule, since OCT (1) is able to identify the lesion activity about 20 days before VA becomes affected, ${ }^{22}$ (2) shows a high agreement with angiographic findings (Table 2), and (3) allows a quantitative analysis of CNV features.

The diagnostic process must include fundus examination, as the appearance of a new hemorrhage could be in itself sufficient to demonstrate $\mathrm{CNV}$ reactivation ${ }^{14}$ and may not always be detectable on OCT. ${ }^{27}$ The LUCAS and SEVENUP trials reported that, at baseline, about a quarter of the study population presented with retinal hemorrhages. ${ }^{20,100}$ Although no data are available regarding the rate of new hemorrhages during follow-up, it is reasonable to hypothesize that retinal bleeding might be present in a small, but significant number of active lesions.

Despite confounding features, SRF normally indicates lesion activity and may be easily detected by OCT. However, if SRF does not decrease after further injections, it should be considered the consequence of chronic RPE decompensation 
or a degenerative phenomenon. ${ }^{57}$ IRF, defined as the presence of intraretinal cysts, nearly always indicates active lesions, with a few exceptions, including degenerative cysts associated with subretinal fibrosis and ORT. ${ }^{41}$

Even if the presence of a PED is not traditionally considered a retreatment criterion, a new onset or an increase in sub-RPE fluid should be introduced in the decision algorithm. Indeed, there have been reports that hollow/serous PEDs respond to anti-VEGF, confirming that it is a probable sign of lesion activity. ${ }^{60}$ Moreover, enlargement of the PED area or volume might serve as a predictor of CNV reactivation, before IRF or SRF appearance. ${ }^{40}$

During the last decade, OCT has progressively become the reference method in follow-up visits, as it is faster and considered to be sufficiently reliable. ${ }^{101}$ Our study group confirmed this clinical practice as strongly supported by literature evidence, and developed a treatment algorithm based on OCT findings, with the exception of clear biomicroscopic findings. Future development of the OCT-A technology will probably improve the precision of this method, to make real-world outcomes much closer to trials' results.

\section{Acknowledgments}

Writing assistance was provided by Dr. Colin Gerard Egan and Dr. Clara Ricci (Primula Multimedia S.r.l.) and funded by Novartis Farma SpA, Italy.

\section{Author Disclosure Statement}

No competing financial interests exist.

\section{References}

1. Klein, R., Klein, B.E., and Cruickshanks, K.J. The prevalence of age-related maculopathy by geographic region and ethnicity. Prog. Retin. Eye Res. 18:371-389, 1999.

2. Wong, T.Y., Wong, T., Chakravarthy, U., et al. The natural history and prognosis of neovascular age-related macular degeneration: a systematic review of the literature and meta-analysis. Ophthalmology. 115:116-126, 2008.

3. Klaver, C.C., Assink, J.J., van Leeuwen, R., et al. Incidence and progression rates of age-related maculopathy: the Rotterdam Study. Invest. Ophthalmol. Vis. Sci.42:2237-2241, 2001.

4. Piermarocchi, S., Segato, T., Scopa, P., et al. The prevalence of age-related macular degeneration in Italy (PAMDI) study: report 1. Ophthalmic. Epidemiol. 18:129-136, 2011.

5. Wong, W.L., Su, X., Li, X., et al. Global prevalence of age-related macular degeneration and disease burden projection for 2020 and 2040: a systematic review and meta-analysis. Lancet. Glob. Health. 2:e106-e116, 2014.

6. Cahill, M.T., Stinnett, S.S., Banks, A.D., Freedman, S.F., and Toth, C.A. Quality of life after macular translocation with 360 degrees peripheral retinectomy for age-related macular degeneration. Ophthalmology. 112:144-151, 2005.

7. Brody, B.L., Gamst, A.C., Williams, R.A., et al. Depression, visual acuity, comorbidity, and disability associated with age-related macular degeneration. Ophthalmology. 108:1893-1900; discuss 1900-1901, 2001.

8. Mitchell, J., and Bradley, C. Quality of life in age-related macular degeneration: a review of the literature. Health Qual. Life Outcomes. 4:97, 2006.

9. Cruess, A.F., Zlateva, G., Xu, X., et al. Economic burden of bilateral neovascular age-related macular degeneration: multi-country observational study. Pharmacoeconomics. 26:57-73, 2008.

10. Soubrane, G., Cruess, A., Lotery, A., et al. Burden and health care resource utilization in neovascular age-related macular degeneration: findings of a multicountry study. Arch. Ophthalmol. 125:1249-1254, 2007.

11. Rosenfeld, P.J., Brown, D.M., Heier, J.S., et al. Ranibizumab for neovascular age-related macular degeneration. N. Engl. J. Med. 355:1419-1431, 2006.

12. Brown, D.M., Michels, M., Kaiser, P.K., et al. Ranibizumab versus verteporfin photodynamic therapy for neovascular age-related macular degeneration: two-year results of the ANCHOR study. Ophthalmology. 116:57.e5-65.e5, 2009.

13. Gillies, M.C., Campain, A., Walton, R., et al. Time to initial clinician-reported inactivation of neovascular agerelated macular degeneration treated primarily with ranibizumab. Ophthalmology. 122:589.e1-594.e1, 2015.

14. Gianniou, C., Dirani, A., Ferrini, W., et al. Two-year outcome of an observe-and-plan regimen for neovascular age-related macular degeneration: how to alleviate the clinical burden with maintained functional results. Eye (Lond.). 29:342-349, 2015.

15. Schmidt-Erfurth, U., Kaiser, P.K., Korobelnik, J.-F., et al. Intravitreal aflibercept injection for neovascular agerelated macular degeneration: ninety-six-week results of the VIEW studies. Ophthalmology. 121:193-201, 2014.

16. CATT Research Group; Martin, D.F., Maguire, M.G., et al. Ranibizumab and bevacizumab for neovascular agerelated macular degeneration. N. Engl. J. Med. 364:18971908, 2011.

17. IVAN Study Investigators; Chakravarthy, U., Harding, S.P., et al. Ranibizumab versus bevacizumab to treat neovascular age-related macular degeneration: one-year findings from the IVAN randomized trial. Ophthalmology. 119:1399-1411, 2012.

18. Kodjikian, L., Souied, E.H., Mimoun, G., et al. Ranibizumab versus bevacizumab for neovascular age-related macular degeneration: results from the GEFAL noninferiority randomized trial. Ophthalmology. 120:2300-2309, 2013.

19. Busbee, B.G., Ho, A.C., Brown, D.M., et al. Twelve-month efficacy and safety of $0.5 \mathrm{mg}$ or $2.0 \mathrm{mg}$ ranibizumab in patients with subfoveal neovascular age-related macular degeneration. Ophthalmology. 120:1046-1056, 2013.

20. Berg, K., Pedersen, T.R., Sandvik, L., and Bragadóttir, R. Comparison of ranibizumab and bevacizumab for neovascular age-related macular degeneration according to LUCAS treatand-extend protocol. Ophthalmology. 122:146-152, 2015.

21. Shah, N., Dakin, S.C., Dobinson, S., Tufail, A., Egan, C.A., and Anderson, R.S. Visual acuity loss in patients with age-related macular degeneration measured using a novel high-pass letter chart. Br. J. Ophthalmol. 100:13461352, 2016.

22. Muether, P.S., Droege, K.M., and Fauser, S. Vascular endothelial growth factor suppression times in patients with diabetic macular oedema treated with ranibizumab. Br. J. Ophthalmol. 98:179-181, 2014.

23. Jones, J., and Hunter, D. Consensus methods for medical and health services research. BMJ. 311:376-380, 1995.

24. Bird, A.C., Bressler, N.M., Bressler, S.B., et al. An international classification and grading system for agerelated maculopathy and age-related macular degeneration. The International ARM Epidemiological Study Group. Surv. Ophthalmol. 39:367-374, 1995.

25. Age-Related Eye Disease Study Research Group. The Age-Related Eye Disease Study system for classifying 
age-related macular degeneration from stereoscopic color fundus photographs: the Age-Related Eye Disease Study Report Number 6. Am. J. Ophthalmol. 132:668-681, 2001.

26. AREDS2 Research Group; Chew, E.Y., Clemons, T., et al. The Age-Related Eye Disease Study 2 (AREDS2): study design and baseline characteristics (AREDS2 report number 1). Ophthalmology. 119:2282-2289, 2012.

27. Amoaku, W.M., Chakravarthy, U., Gale, R., et al. Defining response to anti-VEGF therapies in neovascular AMD. Eye (Lond.). 29:721-731, 2015.

28. Avci, R., Inan, U.U., and Kaderli, B. Long-term results of excision of plaque-like foveal hard exudates in patients with chronic diabetic macular oedema. Eye (Lond.). 22:1099-1104, 2008.

29. Grossniklaus, H.E., and Gass, J.D. Clinicopathologic correlations of surgically excised type 1 and type 2 submacular choroidal neovascular membranes. Am. J. Ophthalmol. 126:59-69, 1998.

30. Klein, M.L., and Wilson, D.J. Clinicopathologic correlation of choroidal and retinal neovascular lesions in agerelated macular degeneration. Am. J. Ophthalmol. 151: 161-169, 2011.

31. Lafaut, B.A., Bartz-Schmidt, K.U., Vanden Broecke, C., Aisenbrey, S., De Laey, J.J., and Heimann, K. Clinicopathological correlation in exudative age related macular degeneration: histological differentiation between classic and occult choroidal neovascularisation. $\mathrm{Br} . \mathrm{J}$. Ophthalmol. 84:239-243, 2000.

32. Ambati, J., Ambati, B.K., Yoo, S.H., Ianchulev, S., and Adamis, A.P. Age-related macular degeneration: etiology, pathogenesis, and therapeutic strategies. Surv. Ophthalmol. 48:257-293, 2003.

33. Rouvas, A.A., Papakostas, T.D., Ntouraki, A., Douvali, M., Vergados, I., and Ladas, I.D. Angiographic and OCT features of retinal angiomatous proliferation. Eye (Lond.). 24:1633-1642; quiz 1643, 2010.

34. Monson, D.M., Smith, J.R., Klein, M.L., and Wilson, D.J. Clinicopathologic correlation of retinal angiomatous proliferation. Arch. Ophthalmol. 126:1664-1668, 2008.

35. Yannuzzi, L.A., Sorenson, J., Spaide, R.F., and Lipson, B. Idiopathic polypoidal choroidal vasculopathy (IPCV). Retina. 10:1-8, 1990.

36. Imamura, Y., Engelbert, M., Iida, T., Freund, K.B., and Yannuzzi, L.A. Polypoidal choroidal vasculopathy: a review. Surv. Ophthalmol. 55:501-515, 2010.

37. Bird, A.C. Doyne Lecture. Pathogenesis of retinal pigment epithelial detachment in the elderly; the relevance of Bruch's membrane change. Eye (Lond.). 5(Pt 1):1-12, 1991.

38. Gass, J.D. Serous retinal pigment epithelial detachment with a notch. A sign of occult choroidal neovascularization. Retina. 4:205-220, 1984.

39. Zayit-Soudry, S., Moroz, I., and Loewenstein, A. Retinal pigment epithelial detachment. Surv. Ophthalmol. 52: 227-243, 2007.

40. Penha, F.M., Gregori, G., Garcia Filho, C.A., Yehoshua, Z., Feuer, W.J., and Rosenfeld, P.J. Quantitative changes in retinal pigment epithelial detachments as a predictor for retreatment with anti-VEGF therapy. Retina. 33:459-466, 2013.

41. Schmidt-Erfurth, U., Chong, V., Loewenstein, A., et al. Guidelines for the management of neovascular age-related macular degeneration by the European Society of Retina Specialists (EURETINA). Br. J. Ophthalmol. 98:11441167, 2014.
42. Schmidt-Erfurth, U., and Waldstein, S.M. A paradigm shift in imaging biomarkers in neovascular age-related macular degeneration. Prog. Retin. Eye Res. 50:1-24, 2016.

43. Koh, A.H.C.; Expert PCV Panel, Chen, L.-J., et al. Polypoidal choroidal vasculopathy: evidence-based guidelines for clinical diagnosis and treatment. Retina. 33:686-716, 2013.

44. Yannuzzi, L.A., Negrão, S., Iida, T., et al. Retinal angiomatous proliferation in age-related macular degeneration. Retina. 21:416-434, 2001.

45. Reche-Frutos, J., Calvo-Gonzalez, C., Pérez-Trigo, S., Fernandez-Perez, C., Donate-Lopez, J., and Garcia-Feijoo, $\mathrm{J}$. Ranibizumab in retinal angiomatous proliferation (RAP): influence of RAP stage on visual outcome. Eur. J. Ophthalmol. 21:783-788, 2011.

46. Parodi, M.B., Iacono, P., Menchini, F., et al. Intravitreal bevacizumab versus ranibizumab for the treatment of retinal angiomatous proliferation. Acta. Ophthalmol. (Copenh). 91:267-273, 2013.

47. Gharbiya, M., Parisi, F., Cruciani, F., Bozzoni-Pantaleoni, F., Pranno, F., and Abdolrahimzadeh, S. Intravitreal antivascular endothelial growth factor for retinal angiomatous proliferation in treatment-naive eyes: long-term functional and anatomical results using a modified PrONTO-style regimen. Retina. 34:298-305, 2014.

48. Yuzawa, M., Mori, R., and Kawamura, A. The origins of polypoidal choroidal vasculopathy. Br. J. Ophthalmol. 89:602-607, 2005.

49. Stanga, P.E., Lim, J.I., and Hamilton, P. Indocyanine green angiography in chorioretinal diseases: indications and interpretation: an evidence-based update. Ophthalmology. 110:15-21; quiz 22-23, 2003.

50. Kwok, A.K.H., Lai, T.Y.Y., Chan, C.W.N., Neoh, E.-L., and Lam, D.S.C. Polypoidal choroidal vasculopathy in Chinese patients. Br. J. Ophthalmol. 86:892-897, 2002.

51. Koh, A., Lee, W.K., Chen, L.-J., et al. EVEREST study: efficacy and safety of verteporfin photodynamic therapy in combination with ranibizumab or alone versus ranibizumab monotherapy in patients with symptomatic macular polypoidal choroidal vasculopathy. Retina. 32:1453-1464, 2012.

52. Tamura, H., Tsujikawa, A., Otani, A., et al. Polypoidal choroidal vasculopathy appearing as classic choroidal neovascularisation on fluorescein angiography. $\mathrm{Br} . J$. Ophthalmol. 91:1152-1159, 2007.

53. Shah, V.P., Shah, S.A., Mrejen, S., and Freund, K.B. Subretinal hyperreflective exudation associated with neovascular age-related macular degeneration. Retina. 34:12811288, 2014.

54. Hogg, R., Curry, E., Muldrew, A., et al. Identification of lesion components that influence visual function in age related macular degeneration. Br. J. Ophthalmol. 87:609614,2003

55. Leuschen, J.N., Schuman, S.G., Winter, K.P., et al. Spectral-domain optical coherence tomography characteristics of intermediate age-related macular degeneration. Ophthalmology. 120:140-150, 2013.

56. Keane, P.A., Patel, P.J., Liakopoulos, S., Heussen, F.M., Sadda, S.R., and Tufail, A. Evaluation of age-related macular degeneration with optical coherence tomography. Surv. Ophthalmol. 57:389-414, 2012.

57. Querques, G., Capuano, V., Frascio, P., Zweifel, S., Georges, A., and Souied, E.H. Wedge-shaped subretinal hyporeflectivity in geographic atrophy. Retina. 35:1735$1742,2015$. 
58. Cohen, S.Y., Dubois, L., Nghiem-Buffet, S., et al. Retinal pseudocysts in age-related geographic atrophy. Am. J. Ophthalmol. 150:211.e1-217.e1, 2010.

59. Zweifel, S.A., Engelbert, M., Laud, K., Margolis, R., Spaide, R.F., and Freund, K.B. Outer retinal tubulation: a novel optical coherence tomography finding. Arch. Ophthalmol. 127:1596-1602, 2009.

60. Punjabi, O.S., Huang, J., Rodriguez, L., Lyon, A.T., Jampol, L.M., and Mirza, R.G. Imaging characteristics of neovascular pigment epithelial detachments and their response to anti-vascular endothelial growth factor therapy. Br. J. Ophthalmol. 97:1024-1031, 2013.

61. Sulzbacher, F., Pollreisz, A., Kaider, A., et al. Identification and clinical role of choroidal neovascularization characteristics based on optical coherence tomography angiography. Acta. Ophthalmol. (Copenh). 95:414-420, 2017.

62. Coscas, G.J., Lupidi, M., Coscas, F., Cagini, C., and Souied, E.H. Optical coherence tomography angiography versus traditional multimodal imaging in assessing the activity of exudative age-related macular degeneration: A new diagnostic challenge. Retina. 35:2219-2228, 2015.

63. El Ameen, A., Cohen, S.Y., Semoun, O., et al. TYPE 2 neovascularization secondary to age-related macular degeneration imaged by optical coherence tomography angiography. Retina. 35:2212-2218, 2015.

64. Kuehlewein, L., Bansal, M., Lenis, T.L., et al. Optical coherence tomography angiography of type 1 neovascularization in age-related macular degeneration. Am. J. Ophthalmol. 160:739.e2-748.e2, 2015.

65. Cole, E.D., Ferrara, D., Novais, E.A., Louzada, R.N., and Waheed, N.K. Clinical trial endpoints for optical coherence tomography angiography in neovascular age-related macular degeneration. Retina. 36(Suppl. 1):S83-S92, 2016.

66. Spaide, R.F. Optical coherence tomography angiography signs of vascular abnormalization with antiangiogenic therapy for choroidal neovascularization. Am. J. Ophthalmol. 160:6-16, 2015.

67. Kuehlewein, L., Sadda, S.R., and Sarraf, D. OCT angiography and sequential quantitative analysis of type 2 neovascularization after ranibizumab therapy. Eye (Lond.). 29:932-935, 2015.

68. Lumbroso, B., Rispoli, M., and Savastano, M.C. Longitudinal optical coherence tomography-angiography study of type 2 naive choroidal neovascularization early response after treatment. Retina. 35:2242-2251, 2015.

69. Tsai, A.S.H., Cheung, N., Gan, A.T.L., et al. Retinal angiomatous proliferation. Surv. Ophthalmol. 62:462-492, 2017.

70. Phasukkijwatana, N., Tan, A.C.S., Chen, X., Freund, K.B., and Sarraf, D. Optical coherence tomography angiography of type 3 neovascularisation in age-related macular degeneration after antiangiogenic therapy. Br. J. Ophthalmol. 101:597-602, 2017.

71. Takayama, K., Ito, Y., Kaneko, H., et al. Comparison of indocyanine green angiography and optical coherence tomographic angiography in polypoidal choroidal vasculopathy. Eye (Lond.). 31:45-52, 2017.

72. Wang, M., Zhou, Y., Gao, S.S., et al. Evaluating polypoidal choroidal vasculopathy with optical coherence tomography angiography. Invest. Ophthalmol. Vis. Sci. 57:OCT526-OCT532, 2016.

73. Mokwa, N.F., Ristau, T., Keane, P.A., Kirchhof, B., Sadda, S.R., and Liakopoulos, S. Grading of age-related macular degeneration: comparison between color fundus photography, fluorescein angiography, and spectral domain optical coherence tomography. J. Ophthalmol. 2013:385915, 2013.

74. Lalwani, G.A., Rosenfeld, P.J., Fung, A.E., et al. A variable-dosing regimen with intravitreal ranibizumab for neovascular age-related macular degeneration: year 2 of the PrONTO study. Am. J. Ophthalmol. 148:43.e1-58.e1, 2009.

75. Chen, E., Kaiser, R.S., and Vander, J.F. Intravitreal bevacizumab for refractory pigment epithelial detachment with occult choroidal neovascularization in age-related macular degeneration. Retina. 27:445-450, 2007.

76. Inoue, M., Arakawa, A., Yamane, S., and Kadonosono, $\mathrm{K}$. Variable response of vascularized pigment epithelial detachments to ranibizumab based on lesion subtypes, including polypoidal choroidal vasculopathy. Retina. 33: 990-997, 2013.

77. Veritti, D., Macor, S., Menchini, F., and Lanzetta, P. Effects of VEGF inhibition on retinal morphology, neovascular network size, and visual acuity in patients with vascularized pigment epithelium detachment because of occult choroidal neovascularization. Retina. 33:982-989, 2013.

78. Castillo, M.M., Mowatt, G., Elders, A., et al. Optical coherence tomography for the monitoring of neovascular age-related macular degeneration: a systematic review. Ophthalmology. 122:399-406, 2015.

79. Truong, S.N., Alam, S., Zawadzki, R.J., et al. High resolution Fourier-domain optical coherence tomography of retinal angiomatous proliferation. Retina. 27:915-925, 2007.

80. Coscas, G., De Benedetto, U., Coscas, F., et al. Hyperreflective dots: a new spectral-domain optical coherence tomography entity for follow-up and prognosis in exudative age-related macular degeneration. Ophthalmologica. 229:32-37, 2013.

81. Tsujikawa, A., Sasahara, M., Otani, A., et al. Pigment epithelial detachment in polypoidal choroidal vasculopathy. Am. J. Ophthalmol. 143:102-111, 2007.

82. Salinas-Alamán, A., García-Layana, A., Maldonado, M.J., Sainz-Gómez, C., and Alvárez- Vidal, A. Using optical coherence tomography to monitor photodynamic therapy in age related macular degeneration. Am. J. Ophthalmol. 140:23-28, 2005.

83. Sandhu, S.S., and Talks, S.J. Correlation of optical coherence tomography, with or without additional colour fundus photography, with stereo fundus fluorescein angiography in diagnosing choroidal neovascular membranes. Br. J. Ophthalmol. 89:967-970, 2005.

84. Eter, N., and Spaide, R.F. Comparison of fluorescein angiography and optical coherence tomography for patients with choroidal neovascularization after photodynamic therapy. Retina. 25:691-696, 2005.

85. Van de Moere, A., Sandhu, S.S., and Talks, S.J. Correlation of optical coherence tomography and fundus fluorescein angiography following photodynamic therapy for choroidal neovascular membranes. Br. J. Ophthalmol. 90:304-306, 2006.

86. van Velthoven, M.E.J., Faber, D.J., Verbraak, F.D., van Leeuwen, T.G., and de Smet, M.D. Recent developments in optical coherence tomography for imaging the retina. Prog. Retin. Eye Res. 26:57-77, 2007.

87. Talks, J., Koshy, Z., and Chatzinikolas, K. Use of optical coherence tomography, fluorescein angiography and in- 
docyanine green angiography in a screening clinic for wet age-related macular degeneration. Br. J. Ophthalmol. 91:600-601, 2007.

88. Malamos, P., Sacu, S., Georgopoulos, M., Kiss, C., Pruente, C., and Schmidt-Erfurth, U. Correlation of highdefinition optical coherence tomography and fluorescein angiography imaging in neovascular macular degeneration. Invest. Ophthalmol. Vis. Sci. 50:4926-4933, 2009.

89. Henschel, A., Spital, G., Lommatzsch, A., and Pauleikhoff, D. Optical coherence tomography in neovascular age related macular degeneration compared to fluorescein angiography and visual acuity. Eur. J. Ophthalmol. 19:831-835, 2009.

90. Khurana, R.N., Dupas, B., and Bressler, N.M. Agreement of time-domain and spectral-domain optical coherence tomography with fluorescein leakage from choroidal neovascularization. Ophthalmology. 117:1376-1380, 2010.

91. Giani, A., Luiselli, C., Esmaili, D.D., et al. Spectraldomain optical coherence tomography as an indicator of fluorescein angiography leakage from choroidal neovascularization. Invest. Ophthalmol. Vis. Sci. 52:55795586, 2011.

92. Padnick-Silver, L., Weinberg, A.B., Lafranco, F.P., and Macsai, M.S. Pilot study for the detection of early exudative age-related macular degeneration with optical coherence tomography. Retina. 32:1045-1056, 2012.

93. Do, D.V., Gower, E.W., Cassard, S.D., et al. Detection of new-onset choroidal neovascularization using optical coherence tomography: the AMD DOC Study. Ophthalmology. 119:771-778, 2012.

94. Wilde, C., Patel, M., Lakshmanan, A., et al. The diagnostic accuracy of spectral-domain optical coherence tomography for neovascular age-related macular degeneration: a comparison with fundus fluorescein angiography. Eye (Lond.). 29:602-609; quiz 610, 2015.

95. de Carlo, T.E., Bonini Filho, M.A., Chin, A.T., et al. Spectral-domain optical coherence tomography angiography of choroidal neovascularization. Ophthalmology. 122:1228-1238, 2015.

96. Gong, J., Yu, S., Gong, Y., Wang, F., and Sun, X. The diagnostic accuracy of optical coherence tomography an- giography for neovascular age-related macular degeneration: a comparison with fundus fluorescein angiography. $J$. Ophthalmol. 2016:7521478, 2016.

97. Tanaka, K., Mori, R., Kawamura, A., Nakashizuka, H., Wakatsuki, Y., and Yuzawa, M. Comparison of OCT angiography and indocyanine green angiographic findings with subtypes of polypoidal choroidal vasculopathy. $\mathrm{Br}$. J. Ophthalmol. 101:51-55, 2017.

98. Inoue, M., Jung, J.J., Balaratnasingam, C., et al. A comparison between optical coherence tomography angiography and fluorescein angiography for the imaging of type 1 neovascularization. Invest. Ophthalmol. Vis. Sci. 57:OCT314-OCT323, 2016.

99. Muether, P.S., Hermann, M.M., Dröge, K., Kirchhof, B., and Fauser, S. Long-term stability of vascular endothelial growth factor suppression time under ranibizumab treatment in age-related macular degeneration. Am. J. Ophthalmol. 156:989.e2-993.e2, 2013.

100. Kuehlewein, L., Dustin, L., Sagong, M., et al. Predictors of macular atrophy detected by fundus autofluorescence in patients with neovascular age-related macular degeneration after long-term ranibizumab treatment. Ophthalmic. Surg. Lasers Imaging Retina. 47:224-231, 2016.

101. Schachat, A.P., and Thompson, J.T. Optical coherence tomography, fluorescein angiography, and the management of neovascular age-related macular degeneration. Ophthalmology. 122:222-223, 2015.

Received: February 16, 2017

Accepted: October 6, 2017

Address correspondence to:

Stefania Miotto, MD

Department of Ophthalmology Camposampiero Hospital ULSS 6 Euganea

Via Gattamelata 134 Padua 35128 Italy

E-mail: stefania.miotto@libero.it 